\title{
EVALUATION OF BIOMASS-DERIVED STABILISING AGENTS FOR COLLOIDAL SILVER NANOPARTICLES VIA NANOPARTICLE TRACKING ANALYSIS (NTA)
}

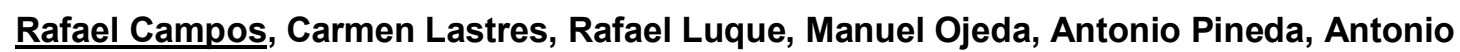 \\ A. Romero, Alfonso Yépez \\ Departamento de Química Orgánica, Universidad de Córdoba, Edifício Marie Curie (C-3), Ctra Nnal IV-A, \\ Km 396, E-14014, Córdoba (Spain), e-mail: campoilus@hotmail.com
}

\begin{abstract}
Nanoparticle Tracking Analysis (NTA) developed by NanoSight has been proved to be a highly useful, simple and efficient characterisation tool to differentiate between capping efficiencies of various biomass-derived stabilising agents (e.g. starch, alginic acid and a waste-derived hemicellulosic syrup) of aqueous colloidal silver suspensions. Results indicated that the use of a complex biorefinery-derived hemicellulosic syrup containing a mixture of $\mathrm{C}_{5}$ and $\mathrm{C}_{6}$ sugars as well as oligomers provided comparable capping and stabilisation properties to those of the most efficient pure polysaccharides including alginic acid. These findings illustrate the potential of waste-derived feedstocks for nanoparticle stabilisation in solution.
\end{abstract}

